\title{
Complex Recanalization of Chronic Total Occluison Supported by Minimal Extracorporeal Circulation in a Patient with an Aortic Valve Bioprothesis in Extraanatomic Position
}

\author{
Ruben Jansen, ${ }^{1,2}$ Brigitte Bathgate, ${ }^{1,2}$ Alexander Bufe, ${ }^{1,2,3}$ and Jan-Erik Guelker $\mathbb{D}^{1,2}$ \\ ${ }^{1}$ Department of Cardiology, Heartcentre Niederrhein, Helios Clinic Krefeld, Krefeld, Germany \\ ${ }^{2}$ Institute for Heart and Circulation Research, University of Cologne, Cologne, Germany \\ ${ }^{3}$ University of Witten/Herdecke, Witten, Germany
}

Correspondence should be addressed to Jan-Erik Guelker; jan-erik.guelker@helios-kliniken.de

Received 12 January 2018; Revised 26 February 2018; Accepted 20 March 2018; Published 5 April 2018

Academic Editor: Man-Hong Jim

Copyright $(2018$ Ruben Jansen et al. This is an open access article distributed under the Creative Commons Attribution License, which permits unrestricted use, distribution, and reproduction in any medium, provided the original work is properly cited.

Percutaneous coronary intervention (PCI) of chronic total occlusion (CTO) still remains a major challenge in interventional cardiology. This case describes a complex PCI of the left main coronary artery and of a CTO of the right coronary artery using a minimal extracorporeal circulation system (MECC) in a patient with an aortic valve bioprothesis in extraanatomic position. It illustrates that complex recanalization strategies can be solved combining it with mechanical circulatory support technologies.

\section{Introduction}

Recanalization of chronic total occlusion (CTO) remains a complex procedure in interventional cardiology. CTO of a coronary artery can be identified in up to $20 \%$ among patients with a clinical indication for coronary angiography. With the advent of novel recanalization techniques and emerging devices, percutaneous coronary interventions (PCIs) have become a promising treatment option for these patients [1-5].

The minimal extracorporeal circulation (MECC) system (Maquet, Hirrlingen, Germany) is a preconnected closed system with a centrifugal pump and a membrane oxygenator. It is a tipto-tip heparin coated system which needs less than $500 \mathrm{ml}$ priming volume. Venous blood returns through active drainage with a centrifugal pump instead of a roller pump to a membrane diffusion oxygenator. Venous reservoir and cardiotomy suction of shed blood is not necessary [6]. We report a case of complex coronary recanalizations using the MECC support in a patient with a calcified coronary artery disease.

\section{Case Report}

We present a case of a 66-year-old woman with advanced coronary artery disease (CAD). In 2013, she underwent coronary artery bypass graft (CABG) surgery with an IMA bypass to the left anterior descending (LAD) and implantation of an aortic valve bioprothesis in extraanatomic position with a conduit from the apex of the left ventricle to the descending aorta for treatment of a symptomatic aortic valve stenosis. This procedure was chosen as a "porcelain" aorta did not allow an implantation in a regular position.

Due to recurrent angina pectoris (CCS III) and dyspnoe (NYHA III), a coronary angiography was performed exhibiting an occlusion of the IMA bypass graft, a high-grade stenosis of the left main coronary artery (LCA) and a chronic total occlusion (CTO) of the right coronary artery (RCA).

Transoesophageal and transthoracal echocardiography revealed a reduced left ventricular function with an ejection fraction of $45 \%$ with mild hypokinesia of the inferior wall. The function of the bioprothesis was regular. This was also confirmed by magnet resonance imaging (MRI) of the heart. The prothesis was positioned $8 \mathrm{~cm}$ distal in the conduit (Figures 1 and 2).

The local heart team decided to perform a percutaneous coronary intervention (PCI) of the left main coronary artery and a PCI of the RCA-CTO assisted by a MECC system.

Before starting the procedure, anesthesia and mechanical ventilation were installed. To prevent thrombembolic 


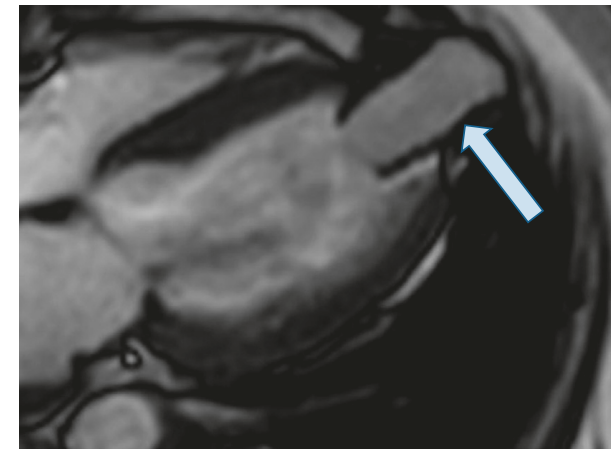

FIgURE 1: MRI shows a conduit from the apex of the left ventricle (arrow).

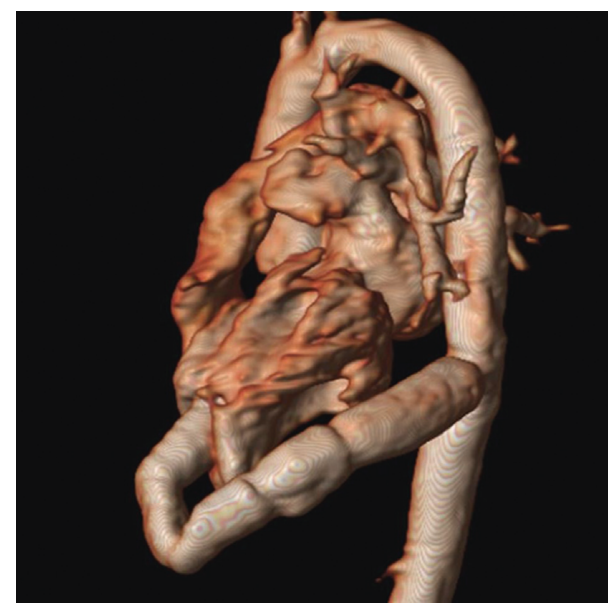

FIGURE 2: MRI shows a conduit from the apex of the left ventricle to the descending aorta.

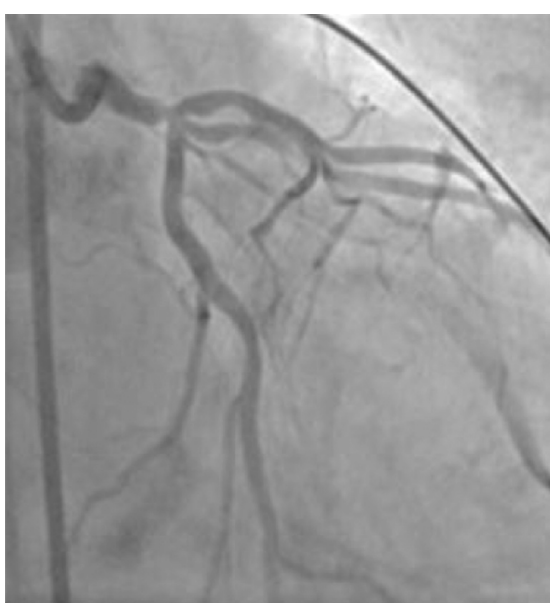

(a)

Figure 3: Continued.

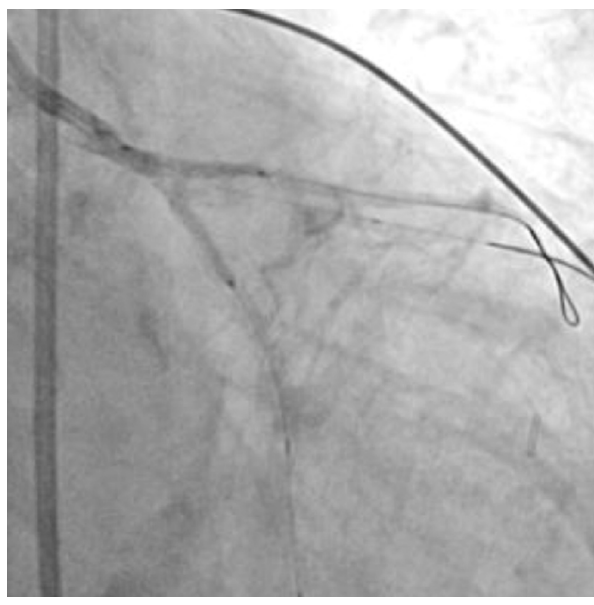

(b)

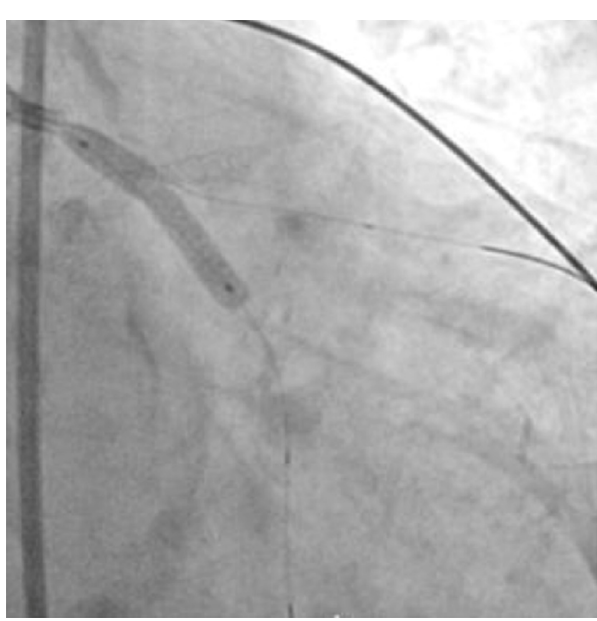

(c)

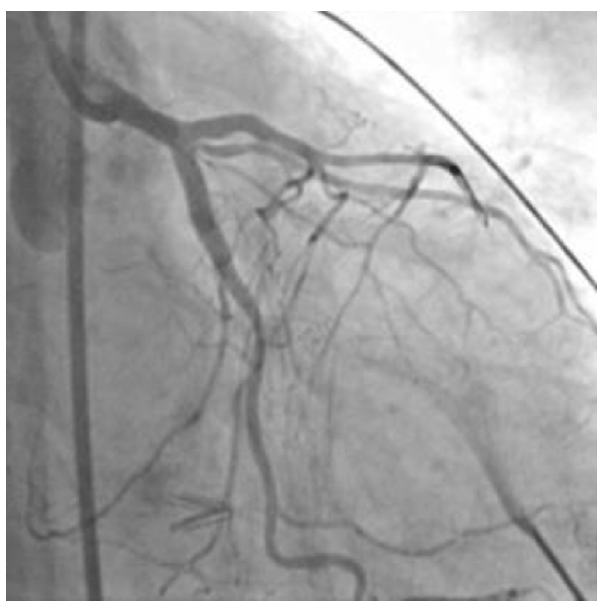

(d)

FIGURE 3: Complex recanalization of the left main coronary artery (a-d). 


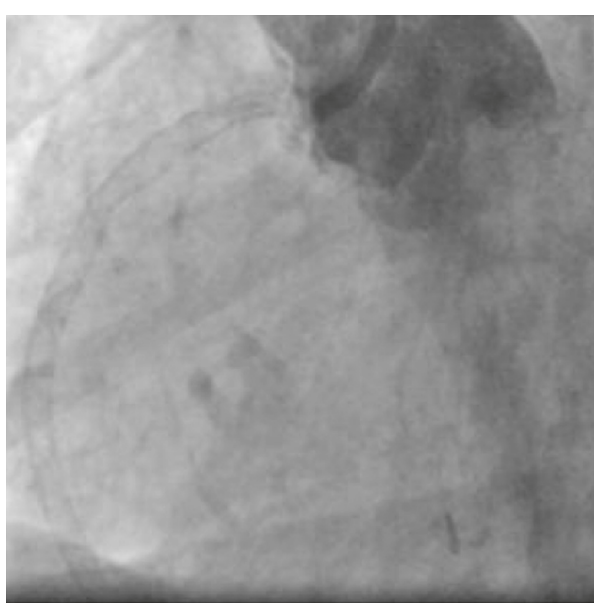

(a)

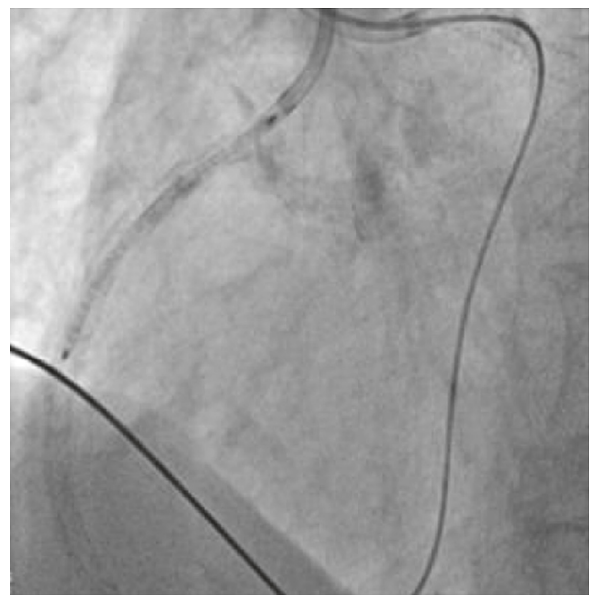

(c)

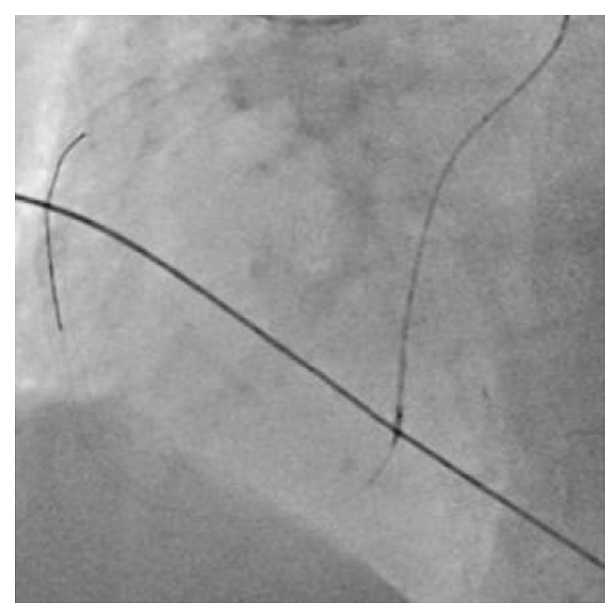

(b)

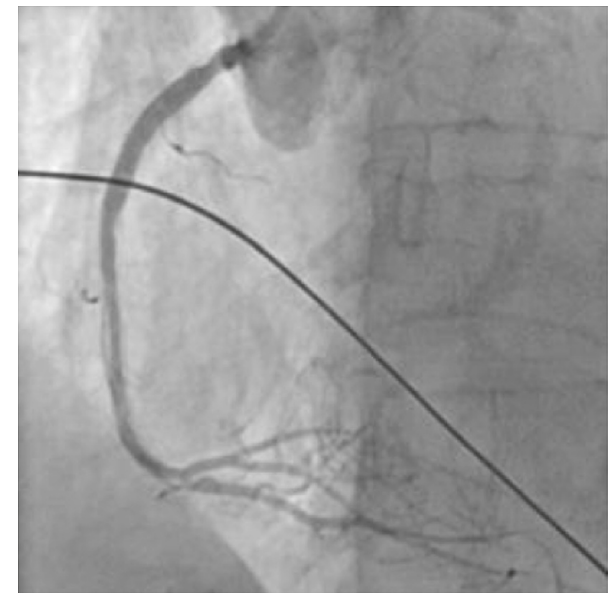

(d)

Figure 4: Retrograde recanalization of the RCA (a-d).

complications, heparin was given during the interventions guided by the activated clotting time $(>300 \mathrm{sec})$. To introduce the minimal extracorporeal circulation system, an arterial 14 French and a venous 21 French access were established. Following a loading dose of $600 \mathrm{mg}$ clopidogrel, a 7F JL4 guiding catheter was advanced to the left main coronary artery, and 0.014" floppy guide wires were introduced into the LAD, left circumflex artery (LCX), and ramus intermedius.

The left main coronary artery was stented in the direction of the LAD and LCX using the mini-crush technique; a $2.75 \times 15 \mathrm{~mm}$ and a $3.5 \times 23 \mathrm{~mm}$ drug-eluting stent (DES) were deployed at 18 and 20 atmospheres, respectively (Figure 3).

After two failed antegrade attempts in the past, the retrograde CTO technique was applied to recanalize the RCA. For recanalization, a Sion wire (Asahi Intecc, Japan) and a Corsair microcatheter (Asahi Intecc, Japan) were used. Wire and microcatheter passed the occluded segment, and after multiple balloon dilatations, a $3.5 \times 38 \mathrm{~mm}$ DES was implanted (Figure 4). After the procedure, we checked the result with intravascular ultrasound (IVUS).
A dual antiplatelet therapy consisting of $100 \mathrm{mg}$ of aspirin once daily and $75 \mathrm{mg}$ of clopidogrel daily for at least 6 months was continued. Artificial ventilation was stopped on the same day, and the patient could leave the hospital five days later.

\section{Discussion}

The MECC system has been introduced in clinical practice in 1999. Initially, it was applied in aortic valve surgery as well as in other cardiac surgical procedures $[7,8]$.

Complex interventional procedures like PCI of the left main or of the CTO often require a hemodynamic support. The advantages of the MECC system in comparison to other mechanical circulatory support technologies like the intraaortic balloon pump (IABP) or the left ventricular assist device (LVAD) are an immediate hemodynamic stabilization and additional support by unloading the right ventricle [9]. As a potential alternative, the Impella CP microaxial pump (Abiomed, USA) has been introduced. Several trials have shown before that cardiac survival was higher in 
patients with complete revascularization when compared to patients with incomplete revascularization $[10,11]$.

Our case demonstrates that by combining advanced recanalization techniques and the MECC system complex, coronary conditions which are so far considered as intractable by interventional means can be treated successfully. The combination of both, high-standard CTO-PCI and an extracorporeal circulation support system, allows us to pursue the interventional procedure. A profound knowledge and a confident command of CTO techniques are required for this kind of intervention. Nevertheless, a strong cooperation between interventional cardiologists and cardiac surgeons is still necessary as part of the heart team [12].

\section{Conflicts of Interest}

The authors declare that there are no conflicts of interest regarding the publication of this article.

\section{References}

[1] P. Fefer, M. L. Knudtson, A. N. Cheema et al., "Current perspectives on coronary chronic total occlusions. The Canadian multicenter chronic total occlusions registry," Journal of the American College of Cardiology, vol. 59, no. 11, pp. 991-997, 2012.

[2] S. D. Tomasello, M. Boukhris, S. Giubilato et al., "Management strategies in patients affected by chronic total occlusions: results from the Italian registry of chronic total occlusions," European Heart Journal, vol. 36, no. 45, pp. 3189-3198, 2015.

[3] A. Bufe, G. Haltern, W. Dinh et al., "Recanalisation of coronary chronic total occlusions with new techniques including the retrograde approach via collaterals," Netherlands Heart Journal, vol. 19, no. 4, pp. 162-167, 2011.

[4] A. R. Galassi, S. D. Tomasello, N. Reifart et al., "In-hospital outcomes of percutaneous coronary intervention in patients with chronic total occlusion: insights from the ERCTO (European registry of chronic total occlusion) registry," EuroIntervention, vol. 7, no. 4, pp. 472-479, 2011.

[5] B. E. Stähli, C. Gebhard, M. Gick et al., "Impact of body mass index on long-term mortality in women and men undergoing percutaneous coronary intervention for chronic total occlusion," International Journal of Cardiology, vol. 224, pp. 305309, 2016.

[6] K. Anastasiadis, P. Antonitsis, A. B. Haidich, H. Argiriadou, A. Deliopoulos, and C. Papakonstantinou, "Use of minimal extracorporeal circulation improves outcome after heart surgery; a systematic review and meta-analysis of randomized controlled trials," International Journal of Cardiology, vol. 164, no. 2, pp. 158-169, 2013.

[7] A. Castiglioni, A. Verzini, F. Pappalardo et al., "Minimally invasive closed circuit versus standard extracorporeal circulation for aortic valve replacement," Annals of Thoracic Surgery, vol. 83, no. 2, pp. 586-591, 2007.

[8] K. Anastasiadis, O. Chalvatzoulis, P. Antonitsis et al., "Use of minimized extracorporeal circulation system in non-coronary and valve cardiac surgical procedures-a case series," Artificial Organs, vol. 35, no. 10, pp. 960-963, 2011.

[9] K. Anastasiadis, S. Hadjimiltiades, and P. Antinitsis, "Successful high-risk percutaneous coronary intervention with the use of minimal extracorporeal circulation system," Catheterization and Cardiovascular Interventions, vol. 80, no. 5, pp. 845-849, 2012.
[10] R. Valenti, A. Migliorini, U. Signorini et al., "Impact of complete revascularization with percutaneous coronary intervention on survival in patients with at least one chronic total occlusion," European Heart Journal, vol. 29, no. 19, pp. 2336-2342, 2008.

[11] Z. Gao, B. Xu, Y. J. Yang et al., "Long-term outcomes of complete versus incomplete revascularization after drug-eluting stent implantation in patients with multivessel coronary disease," Catheterization and Cardiovascular Interventions, vol. 82, no. 3, pp. 343-349, 2013.

[12] P. Kolb, W. Wijns, N. Danchin et al., "Guidelines on myocardial revascularization," European Journal of Cardio-Thoracic Surgery, vol. 38, pp. S1-S52, 2010. 


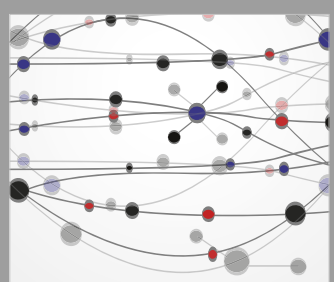

The Scientific World Journal
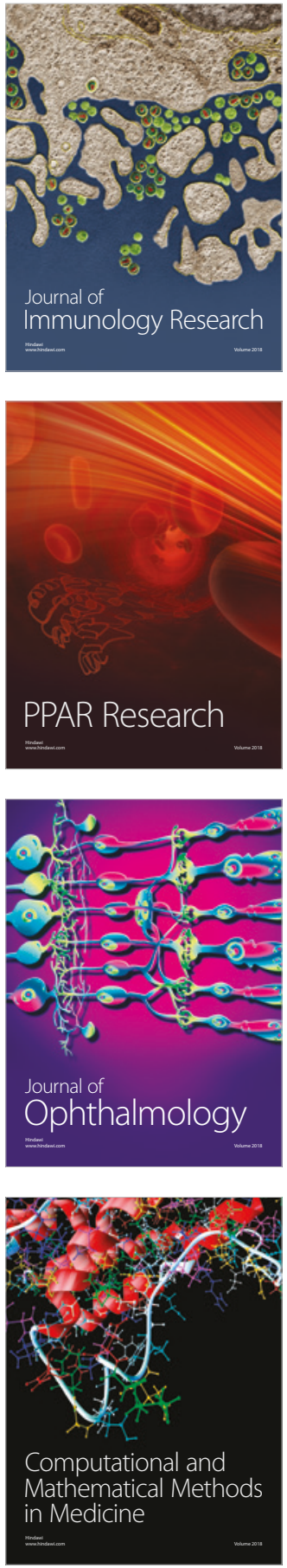

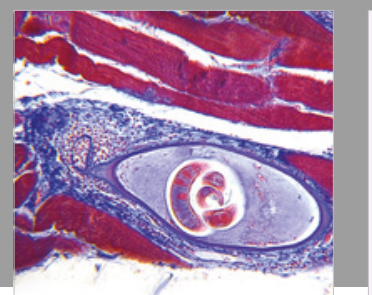

Gastroenterology Research and Practice

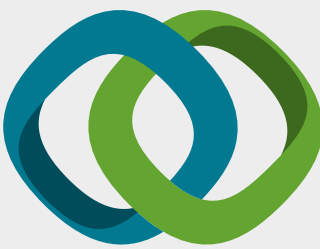

\section{Hindawi}

Submit your manuscripts at

www.hindawi.com
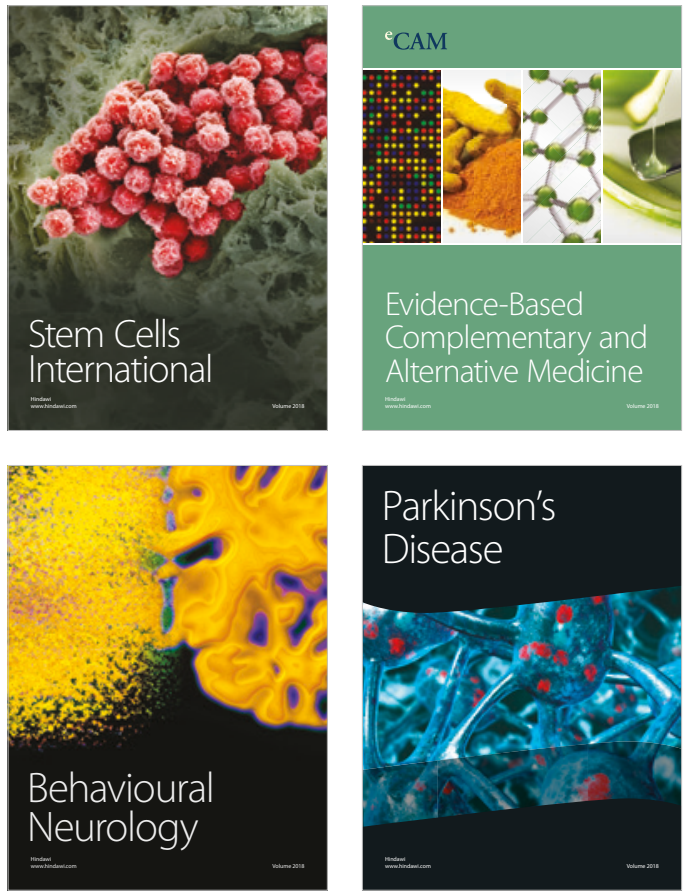

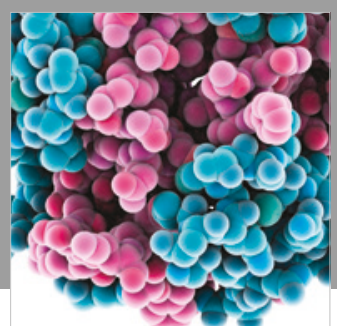

ournal of

Diabetes Research

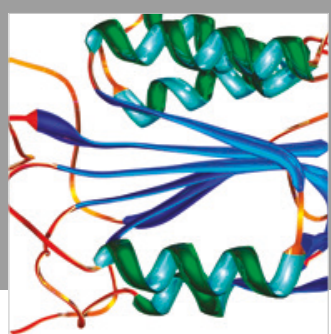

Disease Markers
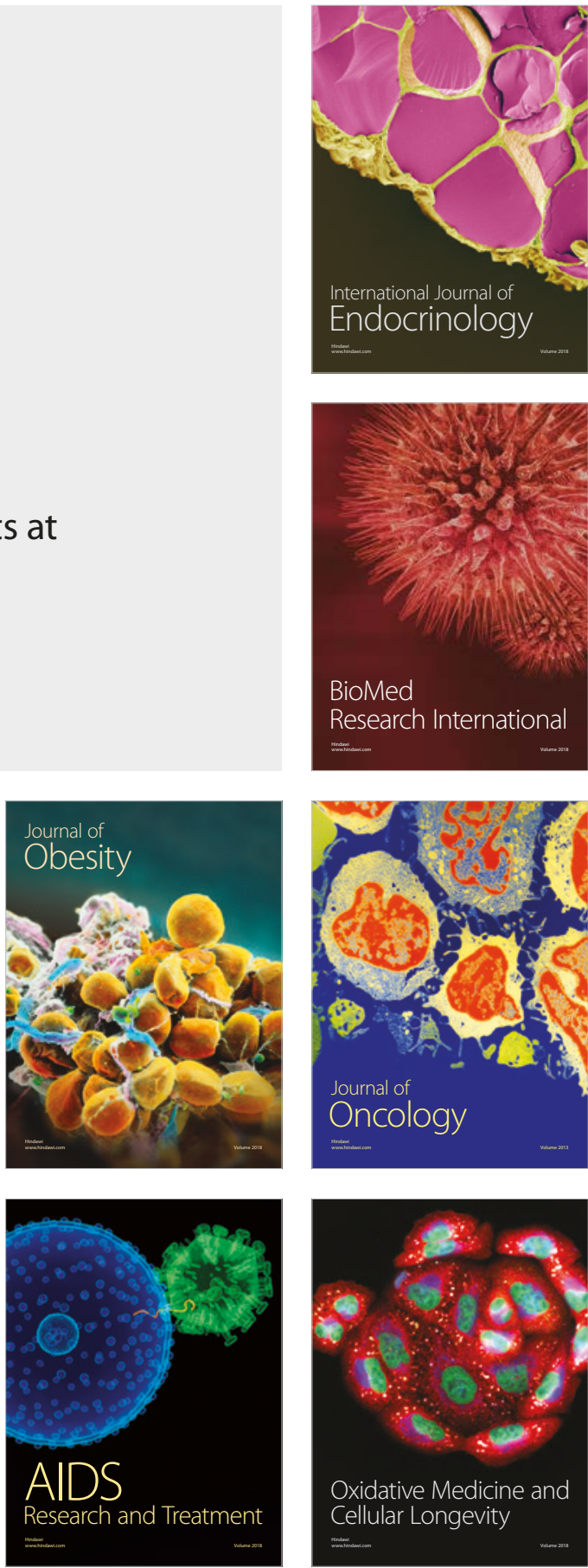